\title{
Analysis of differential expression of long non-coding RNAs in exosomes derived from mature and immature dendritic cells
}

\author{
JIACHANG WU ${ }^{1,2^{*}}$, RENLI ZHAO ${ }^{1,2^{*}}$, JINGXING DAI ${ }^{3}$, GUOHUA LAI $^{1,2}$, ASMAT ULLAH KHAN $^{3}$, \\ XIAO YU ${ }^{4}, \mathrm{SHA} \mathrm{WU}^{4}$, JUN OUYANG ${ }^{3}$ and HONGXUN SANG ${ }^{1,2}$ \\ ${ }^{1}$ Department of Orthopedic Surgery, Shenzhen Hospital of Southern Medical University, Shenzhen, Guangdong 518000; \\ ${ }^{2}$ The Third School of Clinical Medicine, Southern Medical University; \\ ${ }^{3}$ Department of Anatomy, School of Basic Medical Sciences, Southern Medical University, \\ Guangdong Provincial Medical Biomechanical Key Laboratory; ${ }^{4}$ Department of Immunology, \\ School of Basic Medical Sciences, Southern Medical University, Guangzhou, Guangdong 510515, P.R. China
}

Received May 18, 2020; Accepted November 9, 2020

DOI: $10.3892 / \mathrm{mmr} .2020 .11771$

\begin{abstract}
Dendritic cells release bioactive exosomes involved in immune regulation. Long non-coding RNAs (lncRNAs) are implicated in a number of immunoregulatory mechanisms. However, the roles of lncRNAs in dendritic cell-derived exosomes remain to be elucidated. The present study aimed to investigate the roles of lncRNAs in exosomes derived from mature and immature dendritic cells and to find specific lncRNAs with immunoregulatory function. The expression profiles of lncRNAs in exosomes derived from bone marrow dendritic cells of C57 mice were illustrated. Gene Ontology (GO), Kyoto Encyclopedia of Genes and Genomes (KEGG) pathway analyses and Gene Set Enrichment Analysis were performed to identify potential targets correlated with immune regulation. In addition, IncRNA-miRNA-mRNA networks were predicted using bioinformatics methods. Representative lncRNAs were further validated via reverse transcription-quantitative PCR. A total of 437 lncRNAs were analyzed using RNA-seq. Among these, the expression of $\sim 87$ lncRNAs was upregulated and 21 lncRNAs
\end{abstract}

Correspondence to: Professor Hongxun Sang, Department of Orthopedic Surgery, Shenzhen Hospital of Southern Medical University, 1333 Xinhu Road, Shenzhen, Guangdong 518000, P.R. China

E-mail: hxsang@smu.edu.cn

Professor Jun Ouyang, Department of Anatomy, School of Basic Medical Sciences, Southern Medical University, Guangdong Provincial Medical Biomechanical Key Laboratory, 1838 North Guangzhou Avenue, Guangzhou, Guangdong 510515, P.R. China E-mail: jouyang@126.com

*Contributed equally

Key words: dendritic cells, exosome, immunoregulation, long non-coding RNAs was downregulated in mature dendritic cell-derived exosomes (Dex) compared with immature Dex. GO analyses indicated the involvement of upregulated lncRNAs in multiple biological functions, such as the immune system process, while downregulated lncRNAs were involved in poly(A) RNA binding. Analysis of the KEGG pathway identified the relationship of TNF signaling and ribosome pathway with upregulated lncRNAs and downregulated lncRNAs, respectively. The results of gene set enrichment analysis identified that three lncRNA-associated transcripts (Procr-203, Clec4e-202 and Traf1-203) were highly associated with immunoregulatory functions including $\mathrm{T}$ helper cell differentiation and Janus kinase-STAT signaling pathway. The results indicated the involvement of candidate lncRNAs in immunoregulation and suggested a new perspective on the modulation of lncRNAs in Dex.

\section{Introduction}

Dendritic cells (DCs) are antigen-presenting cells that develop directly from myeloid progenitors in the bone marrow and circulating blood monocytes and initiate primary T-cell responses, linking innate and adaptive immune responses (1). DCs have mainly been explored as potent stimulators of adaptive immunity, but DCs also establish and maintain immunological tolerance (2). The state of maturation or activation determines their capacity for initiating tolerance or immunity. Immature (im)DCs induce tolerance, whereas mature (m)DCs induce immunity (3). Some reports have revealed that DCs can prevent, inhibit, or modulate T-cell-mediated effector responses through releasing small $(\sim 40-150 \mathrm{~nm}$ in diameter) membrane-enclosed vesicles (exosomes). These DC-derived exosomes have been proposed to be involved in antigen presentation, immune regulation and signal transduction $(4,5)$.

imDC-derived exosomes (imDex) and $\mathrm{mDC}$-derived exosomes (mDex) have opposite biological functions in regulating the immune network the same as their parental cells $(5,6)$. DC-derived exosomes (Dex) also regulate the cellular behavior of recipient cells following uptake transferring cargo 
molecules. The majority of studies have focused on the function of transmembrane proteins on the surface of imDex and mDex $(6,7)$. Despite the importance of these cargo molecules, only a few studies have paid attention to their intraluminal composition $(8,9)$.

The majority of transcripts transcribed from the human or mouse genome are noncoding (nc)RNAs (10). Among these ncRNAs, long noncoding RNAs (IncRNAs) are >200 nucleotides in length. Numerous lncRNAs have been identified to date and have emerged as significant regulators of various biological processes, such as cell growth, cell activation and metabolic rewiring (11-13). In the context of Dex, the functions of IncRNA remain poorly understood.

In the present study, RNA-sequencing analysis was performed between mDex and imDex. Subsequently, markedly altered biological functions and pathways were predicted by Gene Ontology (GO) and Kyoto Encyclopedia of Genes and Genomes (KEGG) pathway analyses. In addition, the physiological and pathological functions of differentially expressed lncRNAs were identified via the annotation of their associated genes. Finally, the differential expression of representative lncRNAs was further validated by reverse transcription-quantitative (RT-q) PCR. Probable novel mechanisms were identified via a combination of bioinformatics methods. The study of lncRNAs might shed light on the different functions of two kinds of exosomes.

\section{Materials and methods}

Animals and reagents. A total of 50 five-week-old male C57 mice (Animal Center of Southern Medical University, Guangzhou, China), weighing 25-30 g, were used in the present study. All mice were were acclimatized for 1 week before experiments at room temperature under a controlled 12/12 h light/dark cycle and received food and water ad libitum. Antibodies for western blot analysis were as follows: Rabbit monoclonal anti-mouse CD63 (cat. no. ab217345; Abcam), CD9 (cat. no. ab92726; Abcam), CD81 (cat. no. ab109201; Abcam) and TSG101 (cat. no. ab125011; Abcam). Antibodies for flow cytometry were as follows: FITC anti-mouse CD11c (clone N418; Biolegend), PE anti-mouse MHC-II (clone 10-3.6; Biolegend), PE anti-mouse CD80 (clone 16-10A1; Biolegend) and APC anti-mouse CD86 (clone GL-1; Biolegend).

All animal-related experiments were performed according to the guidelines of the Care and Use of Laboratory Animals (Ministry of Health, China, 1998) (14). The experiments were approved by the Animal Use Committee of Shenzhen Hospital, Southern Medical University.

Cultivation of bone marrow dendritic cells. Bone marrow dendritic cells (BMDCs) were obtained from C57 mice as previously described $(6,9)$. Bovine EV-depleted medium was obtained by overnight ultracentrifugation of medium (RPMI-1640; Gibco; Thermo Fisher Scientific, Inc.) supplemented with $20 \%$ fetal bovine serum (FBS; Gibo; Thermo Fisher Scientific, Inc.) at $100,000 \times \mathrm{g}\left(4^{\circ} \mathrm{C}\right.$ for $\left.8 \mathrm{~h}\right)$ to eliminate the interference of exosomes from FBS (9). Briefly, the mice were sacrificed by cervical dislocation. Bone marrow progenitors were washed out from long bones (femur and tibia) and cultured $\left(37^{\circ} \mathrm{C}, 48 \mathrm{~h}\right)$ in EV-depleted medium (10\% EV-depleted
FBS final) containing $20 \mathrm{ng} / \mathrm{ml}$ granulocyte-macrophage colony-stimulating factor (GM-CSF; PeproTech, Inc.) and 10 ng/ml IL-4 (PeproTech, Inc.). Non-adherent cells were gently washed out after $48 \mathrm{~h}$. The remaining clusters, which were loosely adhered to the Petri dish, were cultured $\left(37^{\circ} \mathrm{C}\right.$, 4 days) and the medium was changed every other day. On day 7 , cells were directly observed under a light microscope (magnification, x40 and x100). A total of 6 plates (4 fields of view/plate) were selected to observe cells. Then, cells were collected for treatment and treated with different protocols depending on different studies subsequently conducted.

For exosome isolation, DCs were treated with lipopolysaccharide (LPS; $5 \mu \mathrm{g} / \mathrm{ml}$; Enzo Life Sciences, Inc.) or PBS for $24 \mathrm{~h}$, washed twice with PBS and replaced with fresh medium. After another $48 \mathrm{~h}$ of continuous culture, the culture medium was collected for exosome isolation.

BMDC analysis by flow cytometry. BMDCs were analyzed by flow cytometry for surface marker expression. The dendritic cells were stained with antibodies against CD11c-FITC (1:200; cat.no. 117305),MHC-II-PE(1:1,000; cat.no. 116407),CD80-PE (1:300; cat. no. 104707), CD86-PE (1:160; cat. no. 159203) from BioLegend, Inc. for $45 \mathrm{~min}$ at $4^{\circ} \mathrm{C}$. The cells were stained using a Fortessa flow cytometer (BD Biosciences) and data were analyzed with FlowJo V10 software (FlowJo, LLC).

Exosome isolation and analysis. Exosomes were isolated by differential ultracentrifugation as previously described $(6,9)$. Briefly, the culture medium collected using the aforementioned protocol was centrifuged at $300 \mathrm{x}$ g for $10 \mathrm{~min}$ at $4^{\circ} \mathrm{C}$ to obtain the pellet. The supernatant was centrifuged at 2,000 $\mathrm{x} g$ for $20 \mathrm{~min}$, transferred to new tubes and centrifuged for $40 \mathrm{~min}$ at $10,000 \mathrm{x}$ g and finally for $90 \mathrm{~min}$ at $100,000 \mathrm{x} \mathrm{g}$ at $4^{\circ} \mathrm{C}$. All pellets were washed in 50-60 $\mathrm{ml}$ of PBS and recentrifuged at $100,000 \mathrm{x} \mathrm{g}$ for $90 \mathrm{~min}$ at $4^{\circ} \mathrm{C}$ before being resuspended in 50-100 $\mu 1$ of sterile PBS.

The ultrastructure and size distribution of exosomes were analyzed by transmission electron microscopy (TEM; Hitachi, Ltd.) and NanoSight NS300 (Malvern Panalytical), respectively. Briefly, exosome samples were fixed with $1 \%$ glutaraldehyde in PBS at an optimal concentration at room temperature for $5 \mathrm{~min}$. The mixture was then spotted onto 300-mesh carbon/formvar-coated grids and dried at room temperature. Next, the grids were washed with PBS and stained for contrast using uranyl acetate $(50 \%)$ in water at room temperature for $10 \mathrm{~min}$. Then, exosome size and morphology were observed using a JEM-1011 electron microscope (magnification, x25,000; JEOL Ltd.).

Western blotting. Protein markers, CD63, CD9, TSG101 and CD81 were detected using western blot analysis. The collected exosomes were dissected to extract total protein using RIPA buffer (Applygen Technologies, Inc.) and quantified using a BCA assay. Following quantification, equal amount of proteins $(5 \mu \mathrm{g})$ were separated by $10 \%$ SDS-PAGE and transferred onto PVDF membranes (EMD Millipore).

Following blocking with $5 \%$ skimmed milk at room temperature for $1 \mathrm{~h}$, the membranes were incubated with the following primary antibodies at $4^{\circ} \mathrm{C}$ overnight (all Abcam): CD63 (1:1,000; cat. no. ab217345), CD9 (1:2,000; 
cat. no. ab92726), CD81 (1:3,000; cat. no. ab109201) and TSG101 (1:2,000; cat. no. ab125011). The antibodies were detected using horseradish peroxidase-conjugated IgG (goat anti-rabbit; 1:10,000; cat. no. ab205718; Abcam) at room temperature for $2 \mathrm{~h}$ and visualized using enhanced chemiluminescence (Bio-Rad Laboratories, Inc.).

High-throughput sequencing and analysis of lncRNA. Exosomes were extracted from $\mathrm{mDCs}$ and imDCs as described earlier and the exosomal RNA was extracted using TRIzol ${ }^{\circledR}$ (Thermo Fisher Scientific, Inc.). Extracted lncRNA was enriched with oligo(dT) magnetic beads. RNA-sequencing (RNA-seq) libraries were produced and subjected to quality inspection using an Agilent 2100 (Agilent Technologies $\mathrm{GmbH}$ ). The libraries were quantified by RT-qPCR. The samples were sequenced using an Illumina HiSeq 4000 (Illumina, Inc.). All lncRNAs were annotated based on the Ensembl. RNA-seq and subsequent bioinformatics analysis were performed by ShuPu Biotechnology LLC as previously reported (15).

RNA isolation and RT-qPCR validation of lncRNAs. RT-qPCR was performed as reported previously further to verify the differentially expressed identified lncRNAs (16). RNA from exosome samples $(5 \mu \mathrm{g})$ was extracted using TRIzol ${ }^{\circledR}$ (Thermo Fisher Scientific, Inc.) following the manufacturer's protocols.

RNA quantity and quality were measured using a NanoDrop ND-1000 spectrophotometer (NanoDrop Technologies; Thermo Fisher Scientific, Inc.). The RNA $(1 \mu \mathrm{g})$ was reverse-transcribed using a reverse transcript kit (TransGen Biotech Co., Ltd.) following the manufacturer's protocol. RT-qPCR was performed with a PerfectStart Green qPCR SuperMix kit (TransGen Biotech Co., Ltd.) following the manufacturer's protocol. The primers for validated lncRNAs are listed in Table SI. PCR was performed using a final reaction volume of $20 \mu \mathrm{l}$ and the following thermocycling conditions: $5 \mathrm{~min}$ at $95^{\circ} \mathrm{C}$ for denaturation, 40 cycles of $10 \mathrm{sec}$ at $95^{\circ} \mathrm{C}$ for denaturation, $30 \mathrm{sec}$ at $60^{\circ} \mathrm{C}$ for annealing and elongation, and final extension for $10 \mathrm{~min}$ at $72^{\circ} \mathrm{C}$. Amplification was performed with an ABI-7500 machine (Applied Biosystems; Thermo Fisher Scientific, Inc.). The data were analyzed with the SDS relative quantification software (version 2.2.2; Thermo Fisher Scientific, Inc.).The relative fold change was calculated using the $2^{-\triangle \Delta C q}$ method (17). All reactions were performed in triplicate and normalized to the internal control products of GAPDH.

GO and KEGG pathway analysis of selected lncRNAs. GO (geneontology.org) and KEGG (genome.jp/kegg) analyses of differentially expressed lncRNA-associated genes were performed using the online Database for Annotation, Visualization and Integrated Discovery tool $(18,19)$. The top 10 enriched GO terms among the two groups were identified. GO analysis results consisted of 'biological process' (BP), 'cell composition' (CC) and 'molecular function' (MF). The adjusted P-value was also obtained using the Benjamini \& Hochberg method (20) and an adjusted $\mathrm{P}<0.05$ was considered to indicate a statistically significant difference. The pathways associated with IncRNA-targeted mRNAs were identified by KEGG pathway analysis.
Gene set enrichment analysis. Gene set enrichment analysis (GSEA) is a computational method used to determine whether a given gene set has significant differences among different groups, as previously described (21). Briefly speaking, the Subramanian method, using Java 11 software, was used to first calculate the enrichment score (ES), estimate the importance of ES and finally evaluate their importance by adjusting multiple hypothesis tests.

Prediction of lncRNA-miRNA-mRNA interactions. lncRNA-miRNA interaction was predicted using miRanda (score >150; energy <-30) (22). The target genes of selected miRNAs were predicted and analyzed using miRWalk2.0 (23). The results from six different databases, namely, miRWalk, miRanda, miRDB (mirdb.org/), miRNAMap (mirnamap.mbc. nctu.edu.tw/), RNA22 (cm.jefferson.edu/rna22/Precomputed/) and TargetScan (targetscan.org/mamm_31/), were used for analysis. If a gene was predicted to be a target of miRNA in $>3$ databases, the gene was considered as a target of miRNA. Cytoscape 3.7.1 software (24) was used to construct the network.

Statistical analysis. All experimental data were expressed as the mean \pm standard deviation. Statistical analysis of the data was performed with the two-tailed independent-samples Student's $t$ test using GraphPad Prism 5.0 (GraphPad Software, Inc.). One-way analysis of variance followed by Tukey's honestly significant difference test was used to compare differences among groups. $\mathrm{P}<0.05$ was considered to indicate a statistically significant difference.

\section{Results}

Characterization of BMDCs and BMDC-derived exosomes. BMDCs were cultured in complete medium with recombinant mouse GM-CSF (rmGM-CSF) and recombinant mouse IL-4 (rmIL-4), supplemented with $10 \%$ endotoxin-free and EV-depleted FBS. They were directly observed under a microscope on day 7 and the results demonstrated typical aggregate formation (Fig. 1A). Mature markers were also studied by flow cytometry. Following stimulation with LPS $(5 \mu \mathrm{g} / \mathrm{ml})$ for $24 \mathrm{~h}, \mathrm{mDC}$ expressed high levels of MHC class II and co-stimulatory CD80 and CD86 compared with imDCs (Fig. 1B; the original data are given in Fig. S1). These data indicated a successful culture of BMDCs with EV-depleted complete medium.

On day 7 of DC culture, PBS or LPS was added to the medium to generate immature or mature DCs. After $24 \mathrm{~h}$ of activation by PBS or LPS, the culture medium was replaced completely. After continuously culturing DCs for another $48 \mathrm{~h}$, the exosomes were isolated from the culture supernatants of mDCs and imDCs by differential ultracentrifugation. TEM and nanoparticle tracking analysis were used to analyze the ultrastructure and size distribution of exosomes, respectively. TEM results of exosomes revealed the characteristic saucer shape of exosomes (Fig. 1C). Nanoparticle Tracking Analysis demonstrated that the imDex had a narrow size distribution with a mean particle diameter of $91 \mathrm{~nm}$, which was not significantly different in size compared with mDex (Fig. 1E). Additionally, the expression levels of CD9, CD63, CD81 and 

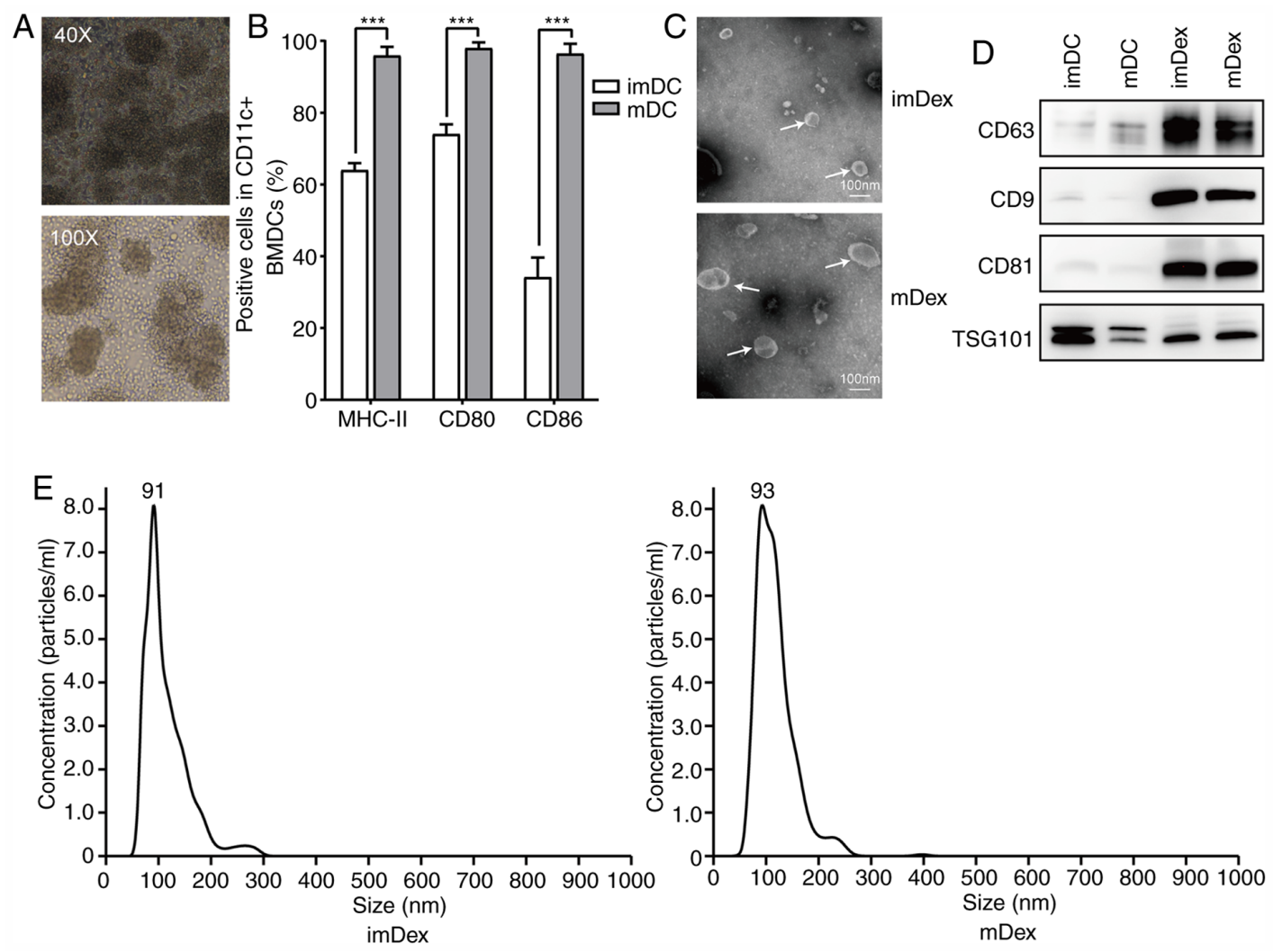

Figure 1. Successful isolation of exosomes from DC culture medium. (A) Morphological structure of BMDCs (magnification, x40 and x100) cultured with previously ultracentrifuged FBS on day 5. (B) Mature markers detected by flow cytometry in imDCs and mDCs cultured with previously ultracentrifuged FBS. ${ }^{* * * *} \mathrm{P}<0.001$. (C) Ultrastructure of mDex and imDex by transmission electron microscopy (white arrows); scale bar, $100 \mathrm{~nm}$. (D) Expression of exosome markers, CD63, CD9, CD81 and TSG101 confirmed by western blot analysis. A total of $5 \mu \mathrm{g}$ protein from DC lysis and $5 \mu \mathrm{g}$ protein from exosomes lysis were loaded onto each lane (representative image of $\mathrm{n}=3$ ). (E) Size distribution profile of mDex and imDex using NanoSight NS300. DC, dendritic cells; BMDCs, bone marrow dendritic cells; im, immature; m, mature; Dex, dendritic cell-derived exosomes.

TSG101 were analyzed by western blot analysis (Fig. 1D). As expected, tetraspanins (CD9, CD63 and CD81) and TSG101 were more abundant in the exosome protein lysis compared with their parental cell protein lysis. These data indicated the successful isolation of exosomes from the culture medium.

Identification of differentially expressed lncRNAs between mDex and imDex. The expression pattern of IncRNAs was detected in three mDex and three imDex samples. A total of 437 lncRNAs were obtained through RNA-seq. Based on the Ensembl (25), 153 lncRNAs were already annotated, whereas 284 lncRNAs were first identified in the present study. Differentially expressed lncRNAs were identified by comparing the differences in the expression levels of these RNAs between mDex and imDex. A total of 108 differentially expressed IncRNAs were analyzed by comparing the differential expression levels. Upregulation was observed in 87 lncRNAs of mDex, whereas downregulation was observed in 21 lncRNAs compared with imDex (Table SII). The heat maps (Fig. 2A), volcano plots at different P-values and fold change (Fig. 2B) and scatter plots (Fig. 2C) were used to show the expression ratios ( $\log 2$ scale) of lncRNAs in mDex and imDex.

Prediction of IncRNA function. GO and KEGG function enrichment analyses for the target genes of differentially expressed lncRNAs were applied to thoroughly understand the functions of the lncRNAs listed in Table SI.
In the $\mathrm{GO}$ analysis, prediction terms with the $\mathrm{P}$-value $<0.05$ were selected. The GO analysis was divided into three parts: $\mathrm{BP}, \mathrm{CC}$ and MF. The top $10 \mathrm{GO}$ terms of three parts associated with the research background were listed. GO analysis clearly revealed that some important biological functions, such as the immune system process and innate immune response, were associated with upregulated lncRNAs (Fig. 3A), whereas the downregulated lncRNAs were mainly involved in the BPs, such as poly(A) RNA binding (Fig. 3B).

In addition, the analysis of the KEGG pathway of lncRNAs of differentially expressed genes demonstrated the relationship of TNF signaling pathway and Toll-like receptor signaling pathway with the upregulated lncRNAs (Fig. 3C), while downregulated lncRNAs were involved in the ribosome pathway (Fig. 3D).

Next, attempts were made to identify features of the top 20 differentially expressed lncRNAs using GSEA. Differential IncRNAs with the same BP and the same pathway were clustered. The biological functions detected using GO analysis, such as T helper cell differentiation, T-cell activation and Janus kinase (JAK)-STAT cascade (Fig. 4A), revealed that some immunoregulatory processes were associated with upregulated IncRNA-associated genes (Procr-203, Clec4e-202 and Traf1-203). The KEGG pathway analysis of the aforementioned three genes revealed JAK-STAT signaling pathway, Th1/Th2 cell differentiation and TNF signaling pathway (Fig. 4C). Cellular components (Fig. S2A) and 

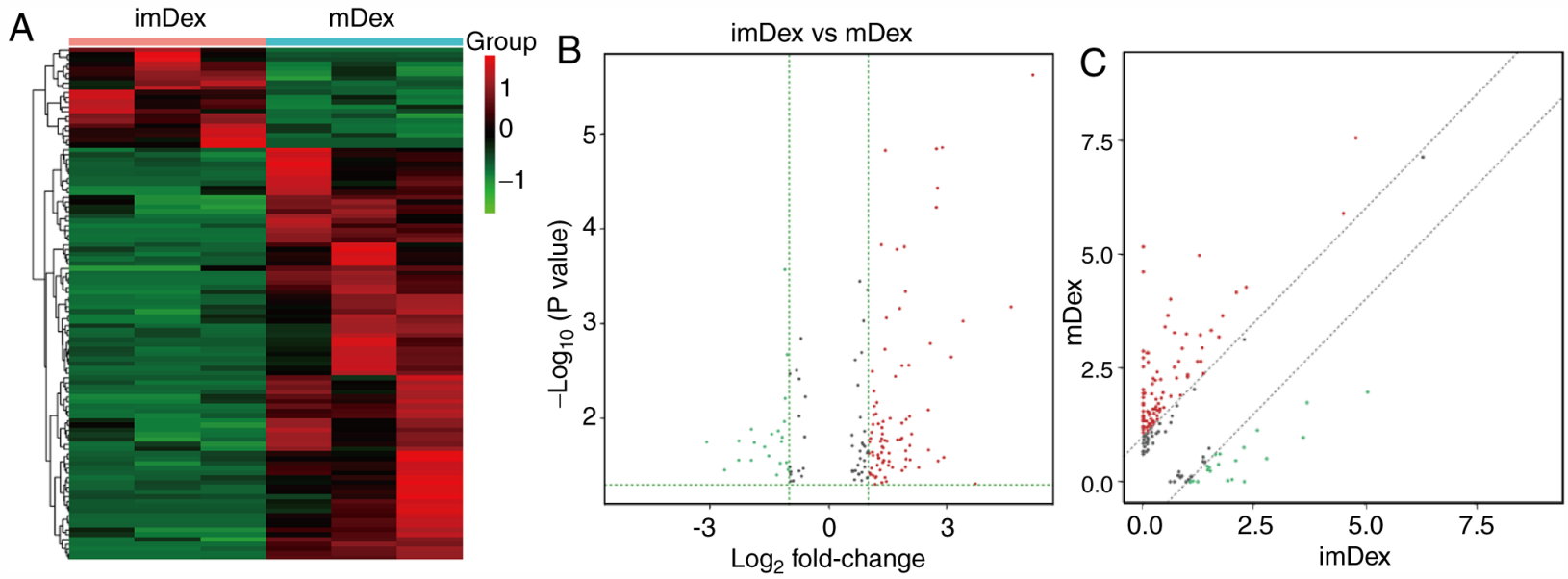

Figure 2. IncRNA expression profile of mDex and imDex. (A) Hierarchical clustering was performed using FPKM values of significantly expressed lncRNAs obtained by comparison between groups, each row representing one lncRNA and each column representing one sample. (B) Volcano plot; the X-axis represents the $\log 2$ fold change value and the y-axis represents - $\log 10$ (P-value). The vertical two green lines are up (right) and down (left) and the green parallel lines correspond to P-value. (C) Scatter plot; the $\mathrm{x}$-axis and $\mathrm{y}$-axis represent the mean FPKM values (log2 transformation) of each group of sample lncRNAs. The two oblique lines divide the upper and lower lncRNAs (1.5-fold difference) and the unmodified lncRNA. lncRNA, long noncoding RNA; m, mature; im, immature; Dex, dendritic cell-derived exosomes; FPKM, Fragments Per Kilobase Million.

molecular functions (Fig. S2B) of GO analysis also provided some clues for lncRNA function annotation.

$R T-q P C R$ validation of the differentially expressed lncRNAs. A total of nine differentially expressed lncRNAs were selected for validation by RT-qPCR to further validate the key differentially expressed lncRNAs identified by RNA-seq. The nine lncRNAs included five upregulated lncRNAs (ENSMUST00000145122, ENSMUST00000143493, ENSMUST00000175954, ENSMUST00000129131 and ENSMUST00000099676) and four downregulated IncRNAs (ENSMUST00000128710, ENSMUST00000129736, ENSMUST00000134860 and ENSMUST00000173666). They were chosen based on the GSEA of their associated genes. These lncRNAs were involved in immunoregulation. The expression levels of verified lncRNAs were consistent with the sequencing results (Fig. 4B).

Identification of IncRNA-targeting miRNAs and construction of the lncRNA-miRNA-mRNA competing endogenous (ce) $R N A$ regulatory network. Based on the GSEA results, five candidates were selected (ENSMUST00000143493, ENSMUST00000175954, ENSMUST00000129131, ENSMUST00000136970 and ENSMUST00000134860) whose functions were most relevant to immunoregulation. An lncRNA-miRNA-mRNA network of the five lncRNAs was constructed (Fig. 4D). First, the target miRNAs of lncRNAs were predicted by choosing the significant correlation pairs according to the score and energy in miRanda. Then, the miRNA-mRNA pairs were predicted using miRWalk2.0 and the top five mRNAs involved in the immunoregulation function were selected and shown in the networks. By integrating the miRNA-mRNA and miRNA-lncRNA regulatory relationships, the miRNA-lncRNA-mRNA network was finally constructed, providing key data for subsequent works.

The resultant network consisted of 14 nodes, including 5 lncRNAs, 16 miRNAs and 75 mRNAs. In total, 98 edges were formed, including 17 lncRNA-miRNA regulation relationships and 81miRNA-mRNA regulation relationships.

\section{Discussion}

Exosomes derived from cell-culture supernatants of dendritic cells are the choice to modulate immune response further in antigen presentation, cancer therapy and a number of other fields in immunology $(26,27)$. In the context of transplantation, allogeneic exosomes from immature DCs can modulate the rejection of heart allografts $(5,28)$. Notably, exosomes derived from tumor peptide-stimulated mature DCs are able to prime tumor-specific cytotoxic $\mathrm{T}$ lymphocyte responses in vivo, resulting in tumor growth delay or the eradication of established murine tumors (29). Dex can serve as cargoes to transfer functional components to $\mathrm{T}$ cells to stimulate their functions (8). Therefore, it was hypothesized in the present study that Dex had different roles in immune networks depending on the state of parental DCs and in part via its intraluminal molecules. lncRNA in exosomes is an important topic, thoroughly studied in types of cancer and a number of other diseases $(30,31)$. However, few studies have explored the physiological or pathological function of lncRNAs in Dex. It was hypothesized that upregulated lncRNAs in different-state DC-derived exosomes may exert different immunoregulatory functions in their recipient cells via vesicle transport through cell-to-cell communication. Thus, the present study hypothesized that immune activation or suppression could, to some extent, be induced by differentially expressed lncRNA in Dex.

The present study investigated the differential expression patterns of lncRNAs in imDex and mDex and predicted the potential functions of selected lncRNAs. A total of 108 differentially expressed lncRNAs (87 upregulated and 21 downregulated) were identified in both imDex and mDex. Some novel findings were deduced by bioinformatics analyses of genes associated with differentially expressed lncRNAs, including identification of the most significantly altered GO categories and KEGG pathways. The KEGG analysis via GSEA demonstrated that the JAK-STAT signaling pathway, reported to be involved in the differentiation of T helper cells, was also associated with upregulated lncRNAs (32). These 

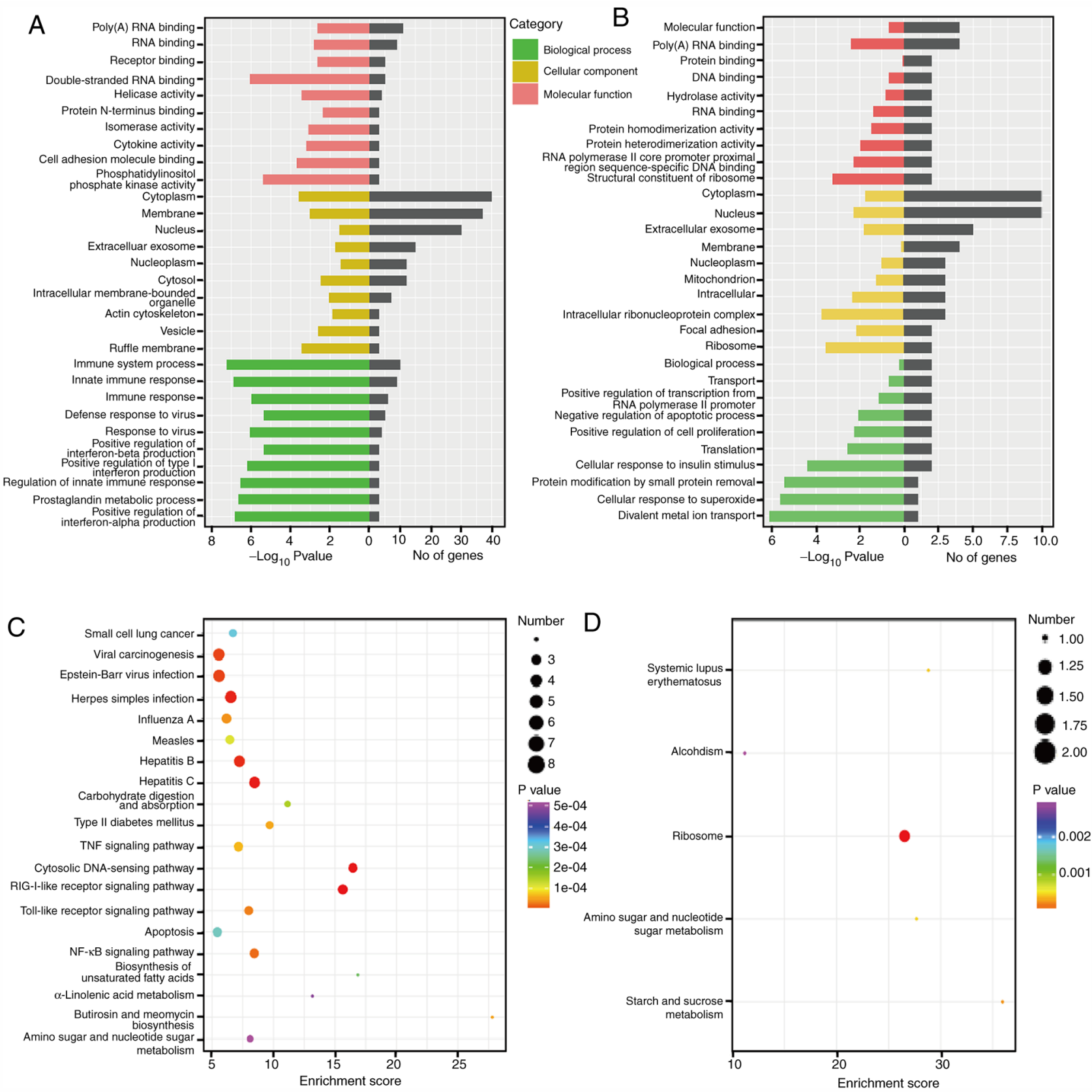

Figure 3. GO enrichment analysis of dysregulated lncRNA gene symbols. (A) Top 10 generally changed GO terms (MF, CC and BP) of significantly upregulated lncRNA gene symbols ranked by [-log10(P-value)] and number of genes. (B) Top 10 generally changed GO terms (MF, CC and BP) of significantly downregulated lncRNA gene symbols ranked by [-log10(P-value)] and number of genes. (C) Bubble chart showing KEGG pathway prediction of target genes of dysregulated lncRNAs. IncRNAs with two-fold change and $\mathrm{P}<0.05$ were selected from the dysregulated lncRNAs and the target genes of these circRNAs were identified using bioinformatics tools. The color of the circle represents the adjusted P-value for each pathway. The size of the circle represents the number of genes enriched. The top 20 pathways of the target genes of upregulated lncRNAs were identified using KEGG analysis. (D) The top 5 pathways of the target genes of downregulated lncRNAs were identified using KEGG analysis. GO, Gene Ontology; IncRNA, long noncoding RNA; MF, molecular function; CC, cell composition; BP, biological process; KEGG, Kyoto Encyclopedia of Genes and Genomes.

finding suggested that the alternation of IncRNA expression could regulate the function of T cells through the JAK-STAT signaling pathway, something which requires further investigation. In addition, nine differentially expressed IncRNAs were selected for further validation using RT-qPCR and the results demonstrated that they were all significantly different.

Based on the GSEA results of the top 10 differentially expressed lncRNAs, the present study focused on five lncRNAs (ENSMUST00000143493, ENSMUST00000175954, ENSMUST00000129131, ENSMUST00000136970 and ENSMUST00000134860) mostly associated with immune-modulating function (33-36). That is also the reason why these five lncRNAs were chosen for further ceRNA network analysis. For instance, protein $\mathrm{C}$ receptor, an associated gene of ENSMUST00000143493, is identified to regulate Th17 pathogenicity via several key molecules of the proinflammatory module in Th17 cells (34). Also, Clec4e, an associated gene of ENSMUST00000175954, is an endocytic receptor expressed in DCs and implicated in corpse scavenging, degradation, or antigen salvage pathways in DC (37). Astudy 
A

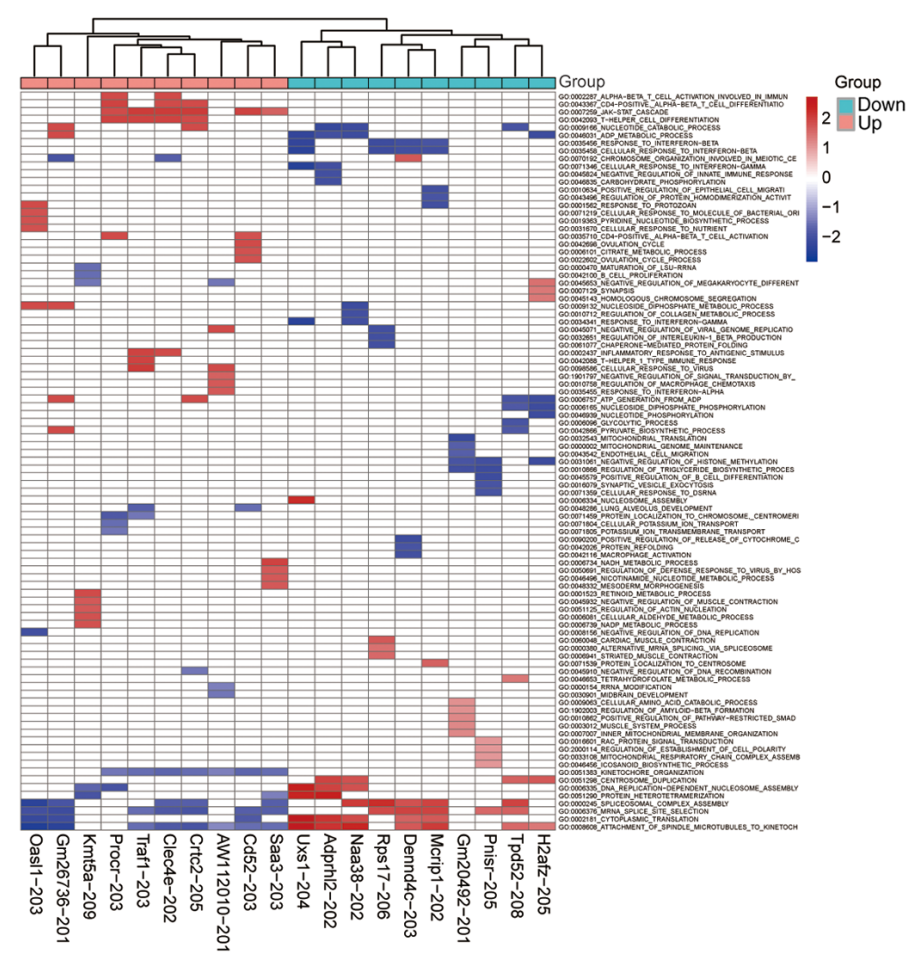

B

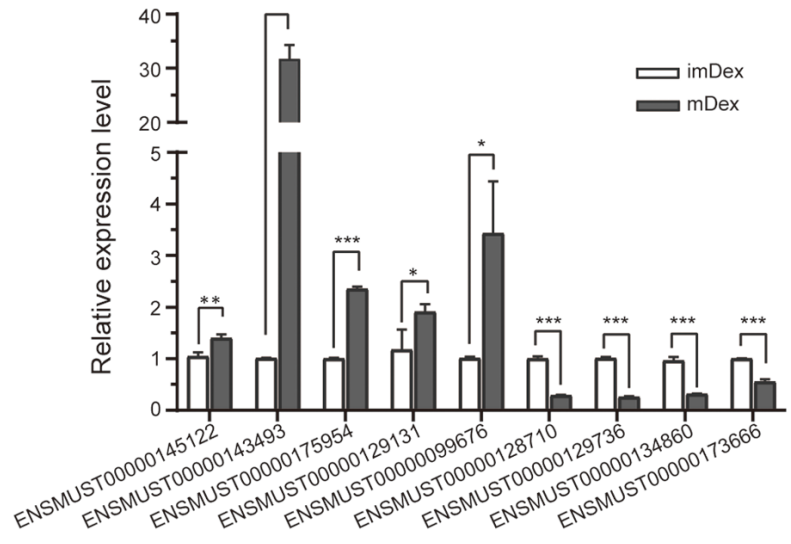

D
C

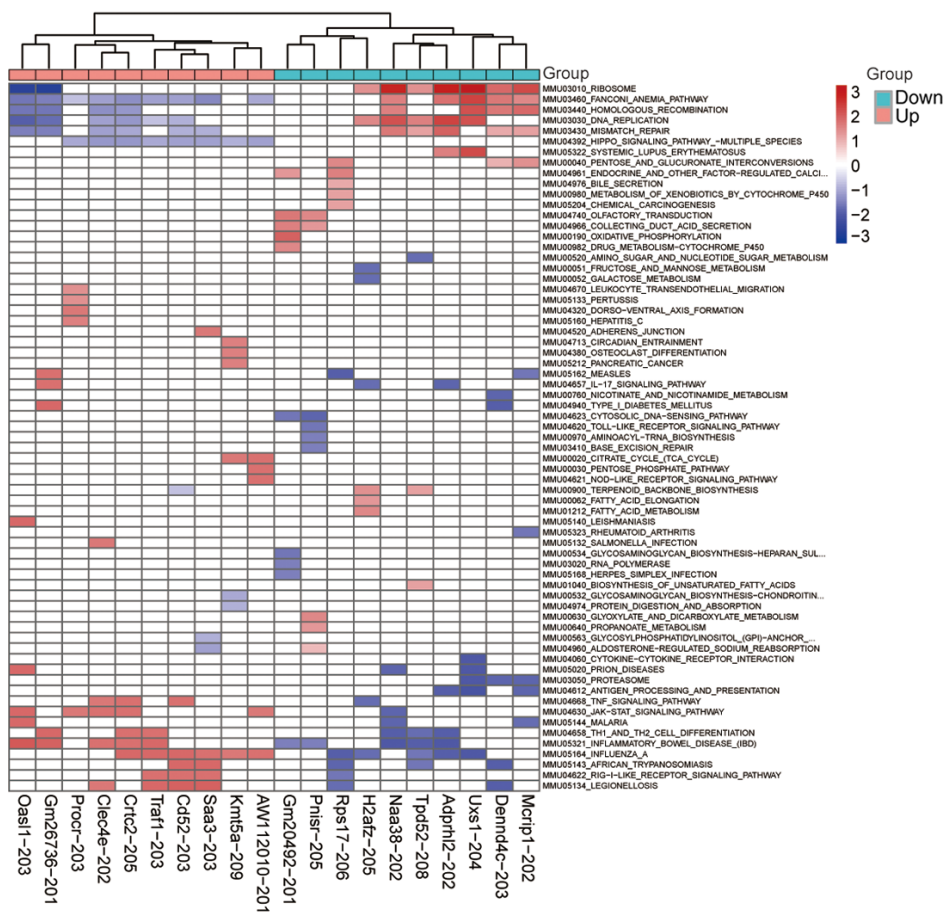

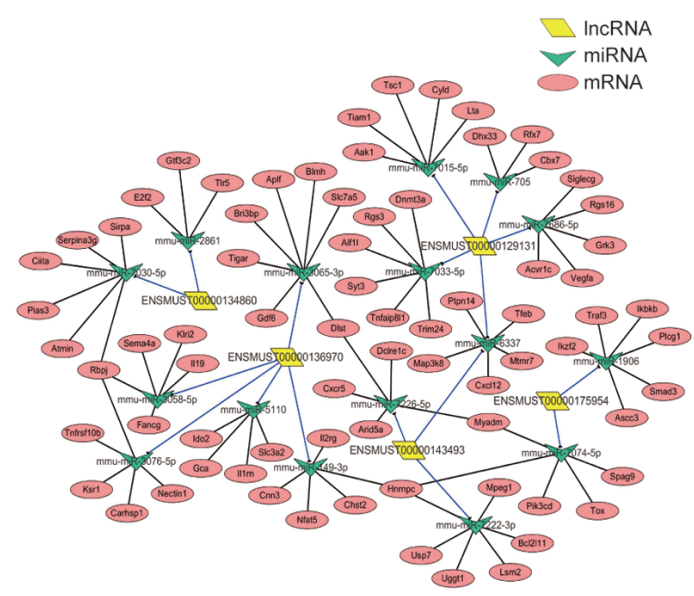

Figure 4. Different lncRNAs GSEA prerank. (A) BP. (B) KEGG pathway; each row represents a functional entry and each column represents an lncRNA. (C) Changes in lncRNA expression were confirmed using reverse transcription-quantitative PCR for selected circRNAs in mDex and imDex groups. Bars represent mean $\pm \operatorname{SEM}\left(\mathrm{n}=3 ;{ }^{*} \mathrm{P}<0.05 ;{ }^{* *} \mathrm{P}<0.01 ;{ }^{* * * *} \mathrm{P}<0.001\right)$. (D) lncRNA candidates $(\mathrm{n}=5)$ were annotated in detail according to the lncRNA/miRNA interaction information using Cytoscape. Based on the miRNA prediction and bioinformatics analyses, mRNAs were found to be regulated by selected miRNAs. Parallelogram, triangles and circles indicate IncRNAs, miRNAs and mRNAs, respectively. IncRNA, long noncoding RNA; GSEA, Gene set enrichment analysis; BP, biological process; KEGG, Kyoto Encyclopedia of Genes and Genomes; circRNA, circular RNA; m, mature; im, immature; Dex, dendritic cell-derived exosomes;

indicated that tumor necrosis factor receptor-associated factor 1 , an associated gene of ENSMUST00000129131, is involved in multiple signaling pathways, including NF- $\mathrm{KB}$ and MAPK pathways (38) and thus influences inflammatory and apoptotic responses to tightly regulate the development of rheumatoid arthritis and chronic infection $(39,40)$. These three lncRNAs, upregulated in $\mathrm{mDex}$, may regulate immune cells via their potential signaling pathways when mDex are taken up by 
recipient cells. However, this hypothesis needs further validation.

Finally, their lncRNA-miRNA-mRNA networks were also constructed using bioinformatics tools for further investigation. All of these miRNAs predicted using miRWalk were associated with the T-cell-receptor signaling pathway. In addition, all the selected targeted genes of miRNAs are reported to regulate the functions of lymphocytes and are enriched in the spleen according to the annotation of PubMed Gene (data not shown) $(41,42)$. On this basis, the present study proposed that the analyzed lncRNAs enriched in different exosomes might participate in various BPs, such as cell activation, differentiation, immune system regulation and some other cellular functions of recipient cells.

In summary, the present study was unveiled lncRNA expression patterns in exosomes derived from dendritic cells in different states, which might help in understanding the role of lncRNAs of exosomes. The present study also indicated the importance of lncRNAs in the imDex- or mDex-modulated immunoregulation.

However, the current study possessed several limitations. First, RNA-seq is an important method to screen possible IncRNAs associated with specific functions and pathways, but the results of big-data analyses may be false positives. Therefore, RT-qPCR should be performed to further verify the differential expression. However, only 9 of these lncRNAs were verified in the present study. Second, the functions of targeted lncRNAs were predicted only indirectly by bioinformatics analysis. Therefore, further functional studies on the mechanism of Dex are warranted to clarify the role of lncRNAs. Finally, due to limited conditions, it was not possible to obtain human cells for the present study, but mouse model can also be helpful for the study of human immune response. For instance, the role of BMDC in modulation of immune response is the same in both human and mice as well as crucial regulatory molecules found in BMDC (43).

\section{Acknowledgements}

Not applicable.

\section{Funding}

The present study was supported by grants from the National Natural Science Foundation of China (grant no. 81672180) and the Sanming Project of Medicine in Shenzhen (grant no. SZSM201612019).

\section{Availability of data and materials}

The data used and/or analyzed in the present study are available from the corresponding author on reasonable request. The raw data (fastq) file has been uploaded to GEO database (GSE156976).

\section{Authors' contributions}

JW and RZ performed the experiments, analyzed the data and drafted the manuscript. JD and GL helped perform the experiments and prepare the materials. XY and SW designed the analytic strategy of the study. AUK, HS and JO designed experiments and edited the manuscript. All authors read and approved the final manuscript.

\section{Ethics approval and consent to participate}

All animal-related experiments were performed according to the guidelines of the Care and Use of Laboratory Animals (Ministry of Health, China, 1998) and the experiments were approved by the Animal Use Committee of Shenzhen Hospital, Southern Medical University.

\section{Patient consent for publication}

Not applicable.

\section{Competing interests}

The authors declare that they have no competing interests.

\section{References}

1. Lu C, Huang X, Zhang X, Roensch K, Cao Q, Nakayama KI, Blazar BR, Zeng Y and Zhou X: miR-221 and miR-155 regulate human dendritic cell development, apoptosis, and IL-12 production through targeting of p27kip1, KPC1, and SOCS-1. Blood 117: 4293-4303, 2011 .

2. Takenaka MC and Quintana FJ: Tolerogenic dendritic cells. Semin Immunopathol 39: 113-120, 2017.

3. Dhodapkar MV, Steinman RM, Krasovsky J, Munz C and Bhardwaj N: Antigen-specific inhibition of effector T cell function in humans after injection of immature dendritic cells. J Exp Med 193: 233-238, 2001.

4. Ma B, Yang JY, Song WJ, Ding R, Zhang ZC, Ji HC, Zhang X, Wang JL, Yang XS, Tao KS, et al: Combining exosomes derived from immature DCs with donor antigen-specific treg cells induces tolerance in a rat liver allograft model. Sci Rep 6: 32971, 2016.

5. Pêche H, Heslan M, Usal C, Amigorena S and Cuturi MC: Presentation of donor major histocompatibility complex antigens by bone marrow dendritic cell-derived exosomes modulates allograft rejection. Transplantation 76: 1503-1510, 2003.

6. Gao W, Liu H, Yuan J, Wu C, Huang D, Ma Y, Zhu J, Ma L, Guo J, Shi H, et al: Exosomes derived from mature dendritic cells increase endothelial inflammation and atherosclerosis via membrane TNF- $\alpha$ mediated NF- $\kappa$ B pathway. J Cell Mol Med 20: 2318-2327, 2016.

7. Thery C, Ostrowski M and Segura E: Membrane vesicles as conveyors of immune responses. Nat Rev Immunol 9: 581-593, 2009.

8. Hao S, Yuan J and Xiang J: Nonspecific CD4(+) T cells with uptake of antigen-specific dendritic cell-released exosomes stimulate antigen-specific CD8(+) CTL responses and long-term T cell memory. J Leukoc Biol 82: 829-838, 2007.

9. Kowal J, Arras G, Colombo M, Jouve M, Morath JP, Primdal-Bengtson B, Dingli F, Loew D, Tkach M and Théry C: Proteomic comparison defines novel markers to characterize heterogeneous populations of extracellular vesicle subtypes. Proc Natl Acad Sci USA 113: E968-E977, 2016.

10. Rinn JL and Chang HY: Genome regulation by long noncoding RNAs. Annu Rev Biochem 81: 145-166, 2012.

11. Militello G, Weirick T, John D, Doring C, Dimmeler S and Uchida S: Screening and validation of lncRNAs and circRNAs as miRNA sponges. Brief Bioinform 18: 780-788, 2017.

12. Fatica A and Bozzoni I: Long non-coding RNAs: New players in cell differentiation and development. Nat Rev Genet 15: 7-21, 2014.

13. Ponting CP, Oliver PL and Reik W: Evolution and functions of long noncoding RNAs. Cell 136: 629-641, 2009.

14. Bao X, Zhang Q, Liu N, Zhuang S, Li Z, Meng Q, Sun H, Bai J, Zhou X and Tang L: Characteristics of circular RNA expression of pulmonary macrophages in mice with sepsis-induced acute lung injury. J Cell Mol Med 23: 7111-7115, 2019. 
15. Luo Z, Mao X and Cui W: Circular RNA expression and circPTPRM promotes proliferation and migration in hepatocellular carcinoma. Med Oncol 36: 86, 2019.

16. Sheng F, Sun N, Ji Y, Ma Y, Ding H, Zhang Q, Yang F and Li W: Aberrant expression of imprinted lncRNA MEG8 causes trophoblast dysfunction and abortion. J Cell Biochem 120: 17378-17390, 2019.

17. Livak KJ and Schmittgen TD: Analysis of relative gene expression data using real-time quantitative PCR and the 2(-Delta Delta C(T)) method. Methods 25: 402-408, 2001.

18. Huang da W, Sherman BT and Lempicki RA: Systematic and integrative analysis of large gene lists using DAVID bioinformatics resources. Nat Protoc 4: 44-57, 2009.

19. Huang da W, Sherman BT and Lempicki RA: Bioinformatics enrichment tools: Paths toward the comprehensive functional analysis of large gene lists. Nucleic Acids Res 37: 1-13, 2009.

20. Xiao B, Zhang W, Chen L, Hang J, Wang L, Zhang R, Liao Y, Chen J, Ma Q, Sun Z and Li L: Analysis of the miRNA-mRNA-lncRNA network in human estrogen receptor-positive and estrogen receptor-negative breast cancer based on TCGA data. Gene 658: 28-35, 2018.

21. An T, Zhang J, Ma Y, Lian J, Wu YX, Lv BH, Ma MH, Meng JH, Zhou YT, Zhang ZY, et al: Relationships of Non-coding RNA with diabetes and depression. Sci Rep 9: 10707, 2019.

22. Enright AJ, John B, Gaul U, Tuschl T, Sander C and Marks DS MicroRNA targets in drosophila. Genome Biol 5: R1, 2003.

23. Dweep H and Gretz N: miRWalk2.0: A comprehensive atlas of microRNA-target interactions. Nat Methods 12: 697, 2015.

24. Shannon P, Markiel A, Ozier O, Baliga NS, Wang JT, Ramage D, Amin N, Schwikowski B and Ideker T: Cytoscape: A software environment for integrated models of biomolecular interaction networks. Genome Res 13: 2498-2504, 2003.

25. Zhang K, Li Q, Kang X, Wang Y and Wang S: Identification and functional characterization of lncRNAs acting as ceRNA involved in the malignant progression of glioblastoma multiforme. Oncol Rep 36: 2911-2925, 2016.

26. Pitt JM, Andre F, Amigorena S, Soria JC, Eggermont A, Kroemer G and Zitvogel L: Dendritic cell-derived exosomes for cancer therapy. J Clin Invest 126: 1224-1232, 2016.

27. Monguio-Tortajada M, Lauzurica-Valdemoros R and Borras FE: Tolerance in organ transplantation: From conventional immunosuppression to extracellular vesicles. Front Immunol 5: 416, 2014

28. Peche H, Renaudin K, Beriou G, Merieau E, Amigorena S and Cuturi MC: Induction of tolerance by exosomes and short-term immunosuppression in a fully MHC-mismatched rat cardiac allograft model. Am J Transplant 6: 1541-1550, 2006.

29. Zitvogel L, Regnault A, Lozier A, Wolfers J, Flament C, Tenza D, Ricciardi-Castagnoli P, Raposo G and Amigorena S: Eradication of established murine tumors using a novel cell-free vaccine: Dendritic cell-derived exosomes. Nat Med 4: 594-600, 1998.

30. Zhu B, Zhang L, Liang C, Liu B, Pan X, Wang Y, Zhang Y, Zhang Y, Xie W, Yan B, et al: Stem cell-derived exosomes prevent aging-induced cardiac dysfunction through a novel exosome/lncRNA MALAT1/NF- $\kappa \mathrm{B} / \mathrm{TNF}-\alpha$ signaling pathway. Oxid Med Cell Longev 2019: 9739258, 2019.
31. Dong H, Wang W, Chen R, Zhang Y,Zou K, Ye M, He X, Zhang F and Han J: Exosome-mediated transfer of lncRNA-SNHG14 promotes trastuzumab chemoresistance in breast cancer. Int J Oncol 53: 1013-1026, 2018.

32. Seif F, Khoshmirsafa M, Aazami H,Mohsenzadegan M,Sedighi G and Bahar M: The role of JAK-STAT signaling pathway and its regulators in the fate of T helper cells. Cell Commun Signal 15: 23, 2017.

33. Pedros C, Altman A and Kong KF: Role of TRAFs in signaling pathways controlling $\mathrm{T}$ follicular helper cell differentiation and $\mathrm{T}$ cell-dependent antibody responses. Front Immunol 9: 2412, 2018.

34. Kishi Y, Kondo T, Xiao S, Yosef N, Gaublomme J, Wu C, Wang C, Chihara N, Regev A, Joller N and Kuchroo VK: Protein C receptor (PROCR) is a negative regulator of Th17 pathogenicity. J Exp Med 213: 2489-2501, 2016.

35. Iborra S, Martínez-López M, Cueto FJ, Conde-Garrosa R, Del Fresno C, Izquierdo HM, Abram CL, Mori D, Campos-Martín Y, Reguera RM, et al: Leishmania uses mincle to target an inhibitory ITAM signaling pathway in dendritic cells that dampens adaptive immunity to infection. Immunity 45: 788-801, 2016.

36. Hernandez JB, Chang C, LeBlanc M, Grimm D, Le Lay J, Kaestner KH, Zheng Y and Montminy M: The CREB/CRTC2 pathway modulates autoimmune disease by promoting Th17 differentiation. Nat Commun 6: 7216, 2015.

37. Yamasaki S, Ishikawa E, Sakuma M, Hara H, Ogata K and Saito T: Mincle is an ITAM-coupled activating receptor that senses damaged cells. Nat Immunol 9: 1179-1188, 2008.

38. Ha H, Han D and Choi Y: TRAF-mediated TNFR-family signaling. Curr Protoc Immunol 11: Unit11 19D, 2009.

39. Wang C, McPherson AJ, Jones RB, Kawamura KS, Lin GHY, Lang PA, Ambagala T, Pellegrini M, Calzascia T, Aidarus N, et al: Loss of the signaling adaptor TRAF1 causes $\mathrm{CD}^{+} \mathrm{T}$ cell dysregulation during human and murine chronic infection. J Exp Med 209: 77-91, 2012.

40. Abdul-Sater AA, Edilova MI, Clouthier DL, Mbanwi A, Kremmer E and Watts TH: The signaling adaptor TRAF1 negatively regulates Toll-like receptor signaling and this underlies its role in rheumatic disease. Nat Immunol 18: 26-35, 2017.

41. Chen Y, Yu M, Zheng Y, Fu G, Xin G, Zhu W, Luo L, Burns R, Li QZ, Dent AL, et al: CXCR5(+)PD-1(+) follicular helper CD8 T cells control B cell tolerance. Nat Commun 10: 4415, 2019.

42. Bishop GA, Stunz LL and Hostager BS: TRAF3 as a multifaceted regulator of B lymphocyte survival and activation. Front Immunol 9: 2161, 2018.

43. Mellor AL and Munn DH: IDO expression by dendritic cells: Tolerance and tryptophan catabolism. Nat Rev Immunol 4: 762-774, 2004

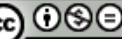

This work is licensed under a Creative Commons Attribution-NonCommercial-NoDerivatives 4.0 International (CC BY-NC-ND 4.0) License. 